\title{
Development and validation of a nomogram to predict anastomotic leakage after esophagectomy for esophageal carcinoma
}

\author{
Wen-Quan Yu, Hui-Jiang Gao", Guo-Dong Shi, Jia-Yu Tang, Hua-Feng Wang, Shi-Yu Hu, Yu-Cheng Wei" \\ Thoracic Surgeon, Department of Thoracic Surgery, The Affiliated Hospital of Qingdao University, Qingdao University, Qingdao, China \\ Contributions: (I) Conception and design: HJ Gao, YC Wei, WQ Yu; (II) Administrative support: WQ Yu; (III) Provision of study materials or \\ patients: WQ Yu, GD Shi; (IV) Collection and assembly of data: WQ Yu; (V) Data analysis and interpretation: WQ Yu, SY Hu; (VI) Manuscript \\ writing: All authors; (VII) Final approval of manuscript: All authors. \\ "These authors contributed equally to this work. \\ Correspondence to: Yu-Cheng Wei, MD. Department of Thoracic Surgery, The Affiliated Hospital of Qingdao University, Qingdao, 266000, China. \\ Email: weiyuchengchest@163.com.
}

\begin{abstract}
Background: This study aimed to identify variables associated with anastomotic leakage after esophagectomy and established a tool for anastomotic leakage prediction.

Methods: Twenty-six preoperative and postoperative variables were retrospectively collected from esophageal cancer patients who were treated with radical esophagectomy from January 2018 to June 2020 in the Affiliated Hospital of Qingdao University. SPSS Version 23.0 and Empower Stats software were used for establishing a nomogram after screening relevant variables by univariate and multivariate Logistic regression analyses. The established nomogram was identified by depicting the receiver operating characteristic (ROC) curves and calibration curve, which was verified by 1,000 bootstrap resamples method.

Results: A total of 604 eligible esophageal cancer patients were included, of which 51 (8.4\%) patients had anastomotic leakage. Multivariate Logistic regression analysis showed that smoking, anastomotic location, anastomotic technique, prognostic nutritional index (PNI) and ASA score were independent risks of anastomotic leakage. The area under curve (AUC) of ROC in the established nomogram was 0.764 (95\% CI, 0.69-0.83). The internal validation confirmed that the nomogram had a great discrimination ability (AUC $=0.766$ ). Depicted calibration curve demonstrated a well-fitted prediction and observation probability. In addition, the decision curve analysis concluded that the newly established nomogram is significant for clinical decision-making.
\end{abstract}

Conclusions: This nomogram provided the individual prediction of anastomotic leakage for esophageal cancer patients after surgery, which might benefit treatment results for patients and clinicians, as well as preand postoperative intervention strategy-making.

Keywords: Esophageal cancer; esophagectomy; anastomotic leakage; prediction model; nomogram

Submitted Jan 31, 2021. Accepted for publication Apr 29, 2021.

doi: $10.21037 /$ jtd-21-209

View this article at: http://dx.doi.org/10.21037/jtd-21-209

\section{Introduction}

Esophageal cancer is the sixth most common cause of cancer-related death in the world. Esophagectomy is the main treatment for resectable patients (1). Anastomotic leakage $(\mathrm{AL})$ is one of the most serious complications after esophagectomy. It is reported that the incidence of AL following esophagectomy ranges 4.9-19.6\% (2,3). AL increases perioperative mortality and recurrence rates, as well as adversely impacts the long-term survival for esophageal cancer patients (4-7). Hence, it is of great 
significance in assessing the risk of postoperative AL in esophageal cancer patients after esophagectomy, which is conductive to recognition and intervention in early period. Previous evidences have identified several risk factors of AL, including comorbidities, American Society of Anesthesiologists (ASA) score, smoking, surgery time, type of operation, anastomotic technique, neoadjuvant chemoradiotherapy, and other preoperative and surgicalrelated variables (8-11).

Scoring systems for assessing surgical risk have been explored in the past, including the well-studied POSSUM and E-PASS system $(12,13)$. Nevertheless, controversies remain in these variables and scoring systems. They are unable to quantify the risk of AL after esophagectomy, which was not conducive to intervention in patients according to risk stratification. Moreover, reliable prediction models and tools for standardized preoperative risk assessment of AL are lacked because of potential influences of multiple variables (4). In this study, we aimed to explore the risk factors of $\mathrm{AL}$ in esophageal cancer patients after esophagectomy and establish a nomogram model for anastomotic leakage prediction, and internal verification was carried out.

We present the following article in accordance with the TRIPOD reporting checklist (available at http://dx.doi. org/10.21037/jtd-21-209).

\section{Methods}

\section{Patient population}

Consecutive esophageal cancer patients who treated with esophagectomy in the Affiliated Hospital of Qingdao University from January 2018 to June 2020 were retrospectively collected. The inclusion criteria were the following: resectable cT1-3/N0-1 esophageal cancer of the middle or lower third of the esophagus and in the gastroesophageal junction (Siewert I), no evidence of distant metastases (including the absence of clinically or histologically confirmed tumor-positive cervical lymph nodes). The exclusion criteria were the following: history of other malignant diseases, previous chest and upper abdominal surgery, pathology is a benign disease of the esophagus, incomplete clinical data, the substitute for the esophagus is not the stomach (jejunum or colon). A total of 604 eligible esophageal cancer patients were included in this study. They were pathologically diagnosed as esophageal cancer. The study was conducted in accordance with the
Declaration of Helsinki (as revised in 2013). The study was approved by the ethics committee of Affiliated Hospital of Qingdao University (NO.: QYFYWZLL26314). Based on the electronic medical record system in our hospital, the clinical data of included patients were completely recorded anonymously. And in this retrospective study, all patients signed the informed consent for operation before the operation, as a result, the informed consent was not needed to be obtained from them again.

\section{Variables}

Based on previous research and clinical experience, we collected baseline characteristics and 26 potential variables relevant to $\mathrm{AL}$, including sex, age, smoking index (smoking index $=$ number of cigarettes per day $\times$ years of smoking), body mass index (BMI), nutritional risk screening (NRS2002), drinking history, ASA score, diabetes history, hypertension history, age-adjusted Charlson comorbidity index (ACCI), neoadjuvant therapy, arrhythmia(Including existing arrhythmia before the operation and new-onset intraoperative cardiac arrhythmia, such as atrial fibrillation, ventricular tachycardia, frequent multisource ventricular premature beats and severe paroxysmal supraventricular tachycardia), tumor location(refer to the definition of tumor location in AJCC 8th Edition esophageal cancer staging Manual), preoperative level of albumin, preoperative level of hemoglobin, preoperative white blood cell count, surgery time, intraoperative blood loss, surgical method (minimally invasive, open or hybrid minimally invasive), anastomotic technique, a switch to thoracotomy, extent of resection, anastomotic location, types of enteral nutrition, pathological type and prognostic nutritional index $(\mathrm{PNI})[\mathrm{PNI}=$ albumin level $(\mathrm{g} / \mathrm{L})+5 \times$ total lymphocyte count $\left.\left(10^{9} / \mathrm{L}\right)\right]$.

\section{Diagnosis and definition of $A L$}

The early symptoms of anastomotic leakage, that is, when the following symptoms occur, should be alert to the occurrence of anastomotic leakage: the presence of redness, induration, and abscess around the anastomosis, a leakage of gas or saliva-like gas (cervical anastomosis) when coughing, drainage of gastric juice, purulent fluid, or bilelike fluid from the chest drainage tube, atrial arrhythmia or unexplained fever in the early stage. Blood routine examination, examinations of $\mathrm{C}$-reactive protein (CRP) and amylase from the thoracic drainage fluid, barium swallow examination (7-10th day after the operation), 
computed tomography (CT; 7-10th day after the operation) were performed in all patients after esophagectomy, and upper gastrointestinal endoscopy was performed only in patients with suspected anastomotic leakage to further confirm the diagnosis (4). Taken together, the diagnosis of anastomotic leakage is comprehensive. Since our overall treatment method is conservative and the patient's hospital stay is relatively long, this study does not include the occurrence of anastomotic leakage after discharge. Based on the definition of AL proposed by the Esophagectomy Complications Consensus Group (ECCG), it was defined as full-thickness defects involving the esophagus, anastomosis, staple line or conduit, irrespective of the presentation or method of identification. AL was specifically classified into three types as follows. Type I was local defects that did not have to change the current therapy, and it did not require medication or dietary modification; Type II was local defects that required interventions (e.g., interventional radiology drain, stent, stent or bedside opening, and packing of incision), rather than surgical procedures; and Type III was local defects that required to be surgically treated (surgical treatment includes: adequate debridement, resection of necrotic tissue, and repair of anastomotic stoma. There was no situation "resection of necrotic conduit" in this study) (14).

\section{Surgical procedures}

After comprehensively evaluating tumor stage, baseline characteristics of esophageal cancer patients, and anatomical characteristics of the cancer, the esophagectomy plan was determined by a multidisciplinary team (MDT). Esophagectomy was done exclusively by six experienced surgeons who specialized in thoracic surgery (>20 surgeries per year). The surgical procedures were depending on the tumor location and the preference of the surgeons. Ivor Lewi, McKeown, or Sweet operations were performed, respectively (15). Briefly, the chest and abdomen were exposed under open surgery or video-assisted thoracoscopic surgery/laparoscopic surgery. Lymphadenectomy (two or three fields) and resection of the cancer part of the esophagus were performed. Subsequently, the stomach was freed using an ultrasonic scalpel, with the preservation of the right gastroepiploic artery. It was then stapled into a tube to make a new esophagus using a linear stapler (16). Thoracic or cervical anastomosis was performed using a circular or liner stapled anastomosis (side to side), or handsewn (end to end). According to the results of preoperative imaging examination, neck lymph node dissection was selectively performed. In this study all case were using gastric tube as a reconstruction substitute, stomach tubes were located in the esophageal bed of the posterior mediastinum.

\section{Statistical analysis}

Data were initially processed using Microsoft Excel 2019, which were further analyzed using SPSS Version 23.0 (Armonk, NY: IBM Corp), EmpowerStats (http:// www.empowerstats.com, $\mathrm{X} \& \mathrm{Y}$ solutions, inc. Boston, Massachusetts) and the R Foundation (http://www.r-project. org; version 3.4.3). The Kolmogorov-Smirnov test was used to evaluate the normal distribution of the continuous variables, and in case of normal distribution, they were presented as mean \pm SD. Continuous variables without normal distribution were presented as median (Q1/4, Q3/4). Categorical variables were presented as frequency and percentage. The comparison between groups of unordered categorical variables was performed using the two-way Chi-square test or Fisher's exact probability test; the comparison between groups of ordered categorical data was performed using the Mann-Whitney U rank-sum test. Recruited patients were divided into groups according to previous experiences and normal medical reference ranges of age, sex, BMI, NRS2002 and hematological parameters. Using SPSS Version 23.0 (Armonk, NY: IBM Corp), patients were divided based on optimal thresholds of smoking index, ACCI, intraoperative blood loss, surgery time and PNI determined by depicting receiver operating characteristic (ROC) curves and calculating Youden index (17). Two-tailed $\mathrm{P}<0.05$ considered as statistically significant. Univariate analysis was performed on all 26 variables. As a result, 9 factors with $\mathrm{P}<0.05$ were found, including: (smoking index, ASA, neoadjuvant therapy, tumor location, surgery time, surgical method, anastomotic technique, extent of resection, anastomotic location, and PNI). These 9 factors were included in the multivariate regression analysis (Forward: Conditional) to determine the predictive factors of anastomotic leakage. Thus, a nomogram and ROC curves were established based on the identified predictive factors using the abovementioned software. Discrimination and calibration of the established nomogram were determined by area under curve (AUC), and calibration curve, respectively (18). Decision Curve Analysis (DCA) was conducted to assess the clinical practicability of the nomogram (19). Finally, the internal 


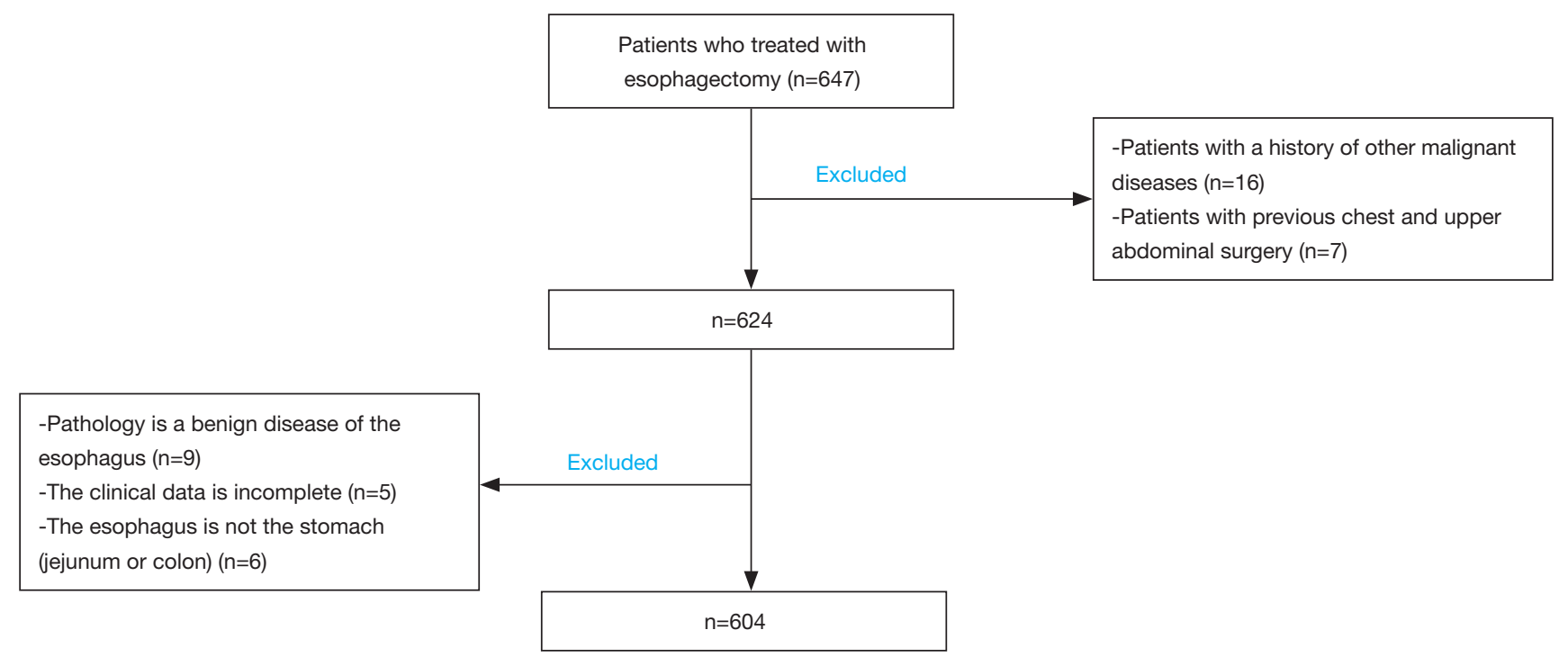

Figure 1 The flow chart of the queue screening process.

validation of the nomogram was performed using 1,000 bootstrap resampling, and AUC after resampling was calculated.

\section{Results}

\section{Patients characteristics}

Searching from the electronic medical record system in our hospital, a total of 604 eligible patients treated with esophagectomy from January 2018 to June 2020 were recruited, involving 564 males and 40 females, with the average age of $63.5 \pm 8.1$ years $(42-87$ years). The flow chart of the queue screening process is shown in Figure 1. Demographic information and clinical data of them were listed in Table 1. A total of 51/604 (8.4\%) cases of $\mathrm{AL}$ occurred after esophagectomy, including 5 (0.8\%) Type I, 41 (6.8\%) Type II and 4 (0.7\%) Type III cases. Among them, a total of 5 patients $(9.8 \%)$ died within 90 days after surgery; and a total of 20 patients (3.8\%) of 533 patients without anastomotic leakage died within 90 days after surgery. The death risk of patients with anastomotic leakage was 3.6 times that of patients without anastomotic leakage (OR 3.65, 95\% CI, 1.28-10.41, $\mathrm{P}<0.05)$. Classified by pathological types, most of recruited esophageal cancer patients were squamous cell carcinoma (479/604, 79.3\%), followed by adenocarcinoma (103/604, $17.1 \%)$. Other types were less, including neuroendocrine carcinoma, undifferentiated carcinoma, mucinous adenocarcinoma and signet-ring cell carcinoma.

\section{Risk factors and prediction factors of $A L$}

The involved 26 variables were subjected to univariate Logistic regression analysis, and 9 were found to be potentially associated with AL, including smoking index, ASA, neoadjuvant therapy, tumor location, surgery time, surgical method, anastomotic technique, extent of resection, anastomotic location, and PNI. Subsequently, the 9 identified variables were further analyzed by introducing them in the multivariate logistic regression model. It is found that cervical anastomotic, high smoking index, high ASA, hand-sewn, and low PNI were independent risk factors of $\mathrm{AL}$ in esophageal cancer patients after esophagectomy $(\mathrm{P}<0.05$; Table 2$)$.

\section{Establishment of the prediction nomogram and validation}

A nomogram based on the five identified risk factors of $\mathrm{AL}$ was established to predict the risk of $\mathrm{AL}$ after esophagectomy (Figure 2). Each patient's five variables will be assigned a score (Table 3), and the corresponding scores of each variable will be added to calculate the individual's total score, so as to obtain the risk of anastomotic leakage. In the original data set, the incidence of anastomotic fistulas with a total score of 130 or less was $7.4 \%$ (37/497), and the incidence of anastomotic fistulas with a total score of 130 to 200 was $11.8 \%$ (11/93). The incidence of anastomotic 
Table 1 Demographic and clinical characteristics of AL group and non-AL Group

\begin{tabular}{|c|c|c|c|c|}
\hline Characteristics & Non-AL $(n=553)$ & $\operatorname{AL}(n=51)$ & Population $(n=604)$ & P-value \\
\hline Female & 37 (6.69) & $3(5.88)$ & $40(6.62)$ & \\
\hline Male & $516(93.31)$ & 48 (94.12) & 564 (93.38) & \\
\hline Age & 64 [57-69] & 66 [58-70] & 64 [57-69] & 0.201 \\
\hline$\geq 65$ & $252(45.57)$ & $28(54.90)$ & $280(46.36)$ & \\
\hline SI & 400 [0-800] & 600 [300-900] & $400[0-800]$ & 0.001 \\
\hline$<400$ & $273(49.37)$ & $13(25.49)$ & $286(47.35)$ & \\
\hline$\geq 400$ & $280(50.63)$ & $38(74.51)$ & $318(52.65)$ & \\
\hline$\geq 18.5-25$ & 385 (69.62) & $28(54.90)$ & $413(68.38)$ & \\
\hline$\geq 25-30$ & $121(21.88)$ & $19(37.25)$ & $140(23.18)$ & \\
\hline$\geq 30$ & $15(2.71)$ & $0(0.00)$ & $15(2.48)$ & \\
\hline NRS2002 & $3[0-4]$ & $3[3-4]$ & $3[0-4]$ & 0.078 \\
\hline$<3$ & $174(31.46)$ & $10(19.61)$ & $184(30.46)$ & \\
\hline$\geq 3$ & 379 (68.54) & 41 (80.39) & $420(69.54)$ & \\
\hline Alcohol consume & & & & 0.237 \\
\hline No drinking history & $197(35.62)$ & $14(27.45)$ & 211 (34.93) & \\
\hline III & $41(7.41)$ & $7(13.73)$ & $48(7.95)$ & \\
\hline Diabetes & & & & 0.292 \\
\hline No & $511(92.41)$ & $45(88.24)$ & $556(92.05)$ & \\
\hline Yes & $42(7.59)$ & $6(11.76)$ & $48(7.95)$ & \\
\hline Hypertension & & & & 0.503 \\
\hline No & $414(74.86)$ & $36(70.59)$ & $450(74.5)$ & \\
\hline Yes & $139(25.14)$ & $15(29.41)$ & $154(25.5)$ & \\
\hline $\mathrm{ACCl}$ & $4[3-5]$ & $4[3-5]$ & $4[3-5]$ & 0.064 \\
\hline$<5$ & $413(74.68)$ & 32 (62.75) & 445 (73.68) & \\
\hline$\geq 5$ & $140(25.32)$ & 19 (37.25) & 159 (26.32) & \\
\hline
\end{tabular}

Table 1 (continued) 
Table 1 (continued)

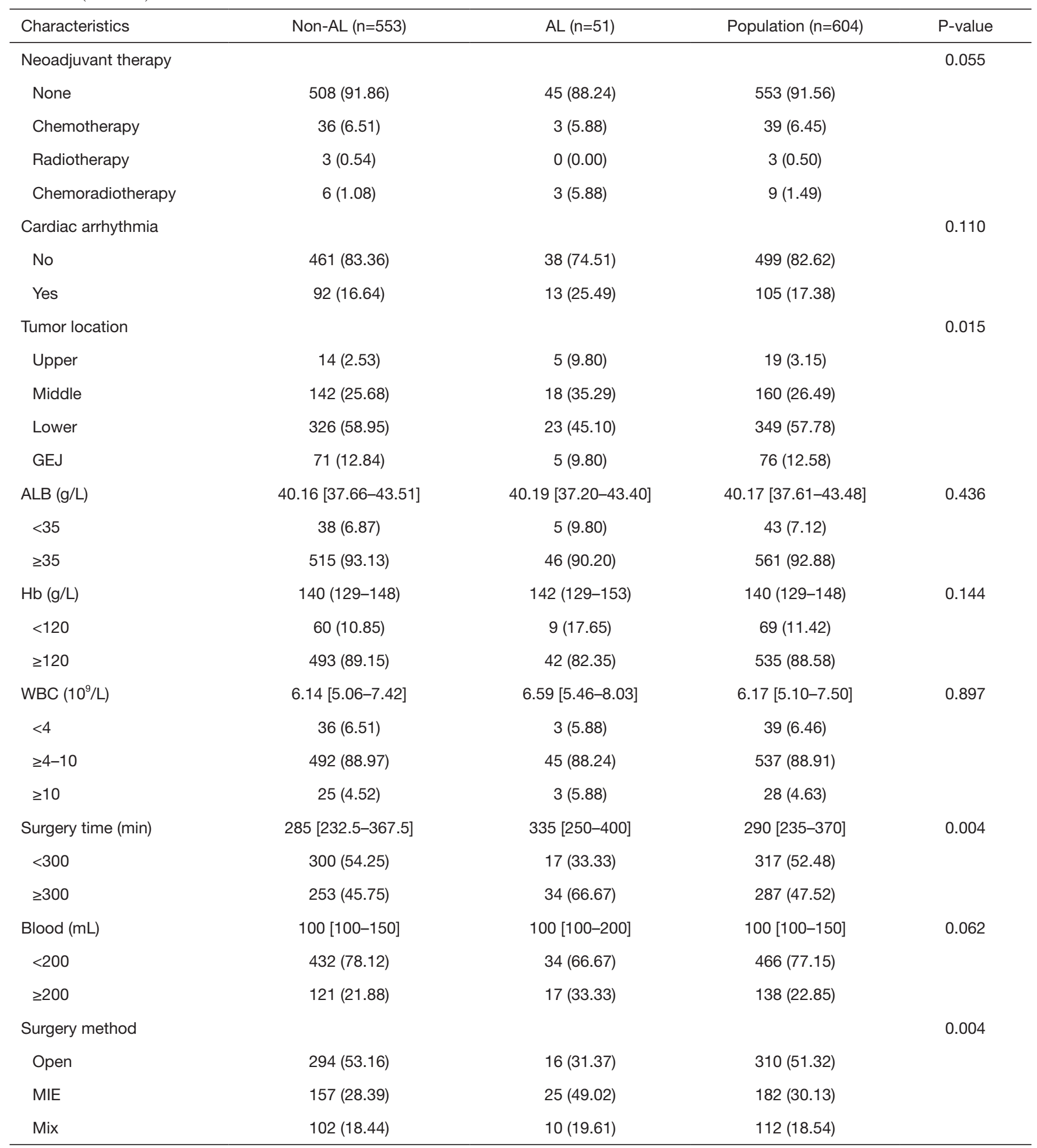

Table 1 (continued) 
Table 1 (continued)

\begin{tabular}{|c|c|c|c|c|}
\hline Characteristics & Non-AL $(n=553)$ & $\mathrm{AL}(\mathrm{n}=51)$ & Population $(n=604)$ & P-value \\
\hline Circular stapled & $476(86.08)$ & 38 (74.51) & $514(85.10)$ & \\
\hline Linear stapled & $71(12.84)$ & $10(19.61)$ & $81(13.41)$ & \\
\hline Hand-sewn & $6(1.08)$ & $3(5.88)$ & $9(1.49)$ & \\
\hline No & $543(98.19)$ & $51(100.00)$ & $594(98.34)$ & \\
\hline Yes & $10(1.81)$ & $0(0.00)$ & $10(1.66)$ & \\
\hline Extent of resection & & & & 0.103 \\
\hline Ro & $532(96.20)$ & $46(90.20)$ & $578(95.70)$ & \\
\hline Anastomosis location & & & & $<0.001$ \\
\hline Chest & $454(82.10)$ & $31(60.78)$ & 485 (80.3) & \\
\hline Neck & 99 (17.90) & 20 (39.22) & $119(19.7)$ & \\
\hline Nutrition mode & & & & 0.012 \\
\hline $\begin{array}{l}\text { Simultaneous nasal- } \\
\text { intestinal tube }\end{array}$ & $228(41.23)$ & $13(25.49)$ & $241(39.9)$ & \\
\hline Simultaneous jejunostomy & $194(35.08)$ & $26(50.98)$ & $220(36.42)$ & \\
\hline $\begin{array}{l}\text { Postoperative nasal- } \\
\text { intestinal canal }\end{array}$ & $92(16.64)$ & $12(23.53)$ & $104(17.22)$ & \\
\hline Other & $19(3.44)$ & $3(5.88)$ & $22(3.65)$ & \\
\hline PNI & $48.84 \pm 5.42$ & $47.05 \pm 4.87$ & $48.70 \pm 5.40$ & 0.005 \\
\hline$<50$ & $335(60.58)$ & $41(80.39)$ & $376(62.25)$ & \\
\hline$\geq 50$ & 218 (39.42) & $10(19.61)$ & $228(37.75)$ & \\
\hline pT-stage & & & & 0.189 \\
\hline 1 & $82(14.82)$ & $5(9.80)$ & $87(14.40)$ & \\
\hline 2 & $118(21.34)$ & $6(11.76)$ & $124(20.50)$ & \\
\hline 3 & $320(57.87)$ & $34(66.67)$ & $354(58.60)$ & \\
\hline 4 & $33(6.00)$ & $6(11.77)$ & $39(6.46)$ & \\
\hline
\end{tabular}

Table 1 (continued) 
Table 1 (continued)

\begin{tabular}{lccc}
\hline Characteristics & Non-AL $(n=553)$ & AL $(n=51)$ & Population (n=604) \\
\hline PN-stage & $252(45.57)$ & $23(45.10)$ & $275(45.53)$ \\
0 & $151(27.31)$ & $13(25.49)$ & $164(27.15)$ \\
1 & $99(17.90)$ & $9(17.65)$ & $108(17.88)$ \\
2 & $51(9.22)$ & $6(11.76)$ & $57(9.44)$ \\
3 & & & 0.518 \\
pTNM-stage & $76(13.74)$ & $4(7.84)$ & $80(13.25)$ \\
1 & $181(32.73)$ & $16(31.73)$ & $197(32.62)$ \\
3 & $236(42.68)$ & $23(45.10)$ & $259(42.88)$ \\
4 & $60(10.85)$ & $8(15.69)$ & $68(11.26)$ \\
\hline
\end{tabular}

Data presented as $\mathrm{n}(\%), M\left[\mathrm{Q}_{1 / 4}-\mathrm{Q}_{3 / 4}\right]$, or mean \pm standard deviation. AL, anastomotic leakage; SI, smoking index; BMI, body mass index; NRS, nutrition risk screening; ASA, American Society of Anesthesiologists; ACCI, age-adjusted Charlson comorbidity index; GEJ, gastroesophageal junction; ALB, albumin; Hb, hemoglobin; WBC, white blood cell; PNI, prognostic nutritional index.

leakage with a total score of 200 and above was $21.4 \%$ (3/14). The probability of anastomotic leakage in different segments is statistically different $(\mathrm{P}=0.048)$. Then, the ROC curve was established to test the discrimination of the model (AUC $=0.764)$ (Figure 3) and the model was verified internally by using 1,000 bootstrap resampling (AUC $=0.766$ ), indicating that the established nomogram has good discrimination in predicting AL (18). Furthermore, the calibration curve suggested a well fitted prediction and observation probability of the nomogram (Figure 4). The DCA was shown in Figure 5, where a red curve represented clinical benefits of patients at different risk levels of AL. which identified that more benefits could be obtained by using the model to identify the anastomotic leakage to take intervention measures than the original treatment strategy.

\section{Discussion}

AL remains to be a severe complication after esophagectomy and independent factor for increasing the risk of postoperative death, although surgical procedures and quality of care have been greatly proved in recent years. It is reported that the risk of death in patients with $\mathrm{AL}$ after esophagectomy is three times higher than whom without $\mathrm{AL}$, which is up to $18 \%$ (6). Similar results were obtained in this study. Besides, AL also influences the longterm prognosis of esophageal cancer patients. Compared with postoperative patients without AL, the overall survival
(35.8 vs. 54.8 months, $\mathrm{P}=0.002$ ) and disease-free survival (34.0 vs. 47.9 months, $\mathrm{P}=0.005$ ) of esophageal cancer patients with AL are significantly lower (7). Previous studies have reported that multiple factors are considered as risk factors of AL, including comorbidities, ASA score, smoking, surgery time, surgical method, anastomotic technique, neoadjuvant therapy, etc. $(4,8,9,20)$. However, these identified factors are unable to quantify the risk of AL. As early in 1991, Copeland et al. analyzed the incidence of complications and 30-day mortality in a total of 1,372 patients requiring surgery during the past 6 months, and established the POSSOM system involving 12 physiological variables and 6 surgical variables for predicting surgical risk (21). Nevertheless, the rationality and accuracy of the POSSOM system are controversial since the data are processed by index analysis, rather than the standard linear analysis. Multiple studies pointed out that the POSSOM system is unable to accurately assess the surgical risk of esophageal cancer. In addition, Haga et al. assessed the function of the E-PASS system in evaluating digestive surgery (13). They proposed that patients with a higher total risk point (TRP) are more prone to develop postoperative AL. However, the E-PASS system has multiple limitations. It does not take into consideration of factors associated with surgical method, and lacks of pertinence in patients treated with esophagectomy and the ability to quantify the risk of postoperative AL. In the present study, we established a nomogram to predict the risk of $\mathrm{AL}$ in esophageal cancer 
Table 2 Univariate and multivariate logistic regression analysis

\begin{tabular}{|c|c|c|c|c|c|c|}
\hline Characteristics & \multicolumn{3}{|c|}{ Univariable } & \multicolumn{3}{|c|}{ Multivariable } \\
\hline \multicolumn{7}{|l|}{ Gender } \\
\hline Female & Reference & & & & & \\
\hline Male & 1.15 & $0.34-3.86$ & 0.824 & & & \\
\hline$<65$ & Reference & & & & & \\
\hline$\geq 65$ & 1.45 & $0.82-2.59$ & 0.203 & & & \\
\hline \multicolumn{7}{|l|}{ SI } \\
\hline$<400$ & Reference & & & Reference & & \\
\hline$<18.5$ & Reference & & & & & \\
\hline$\geq 18.5-25$ & 0.58 & $0.19-1.76$ & 0.338 & & & \\
\hline$\geq 25-30$ & 1.26 & $0.40-3.95$ & 0.697 & & & \\
\hline$\geq 30$ & 0 & - & 0.981 & & & \\
\hline \multicolumn{7}{|l|}{ NRS2002 } \\
\hline$<3$ & Reference & & & & & \\
\hline$\geq 3$ & 1.88 & $0.92-3.84$ & 0.083 & & & \\
\hline \multicolumn{7}{|l|}{ Alcohol consume } \\
\hline II & 1.66 & $0.89-3.11$ & 0.111 & 1.56 & $0.81-3.02$ & 0.185 \\
\hline III & 2.60 & $1.02-6.60$ & $0.045^{\star}$ & 8.21 & $2.72-24.77$ & $<0.001^{*}$ \\
\hline \multicolumn{7}{|l|}{ Diabetes } \\
\hline No & Reference & & & & & \\
\hline Yes & 1.62 & $0.65-4.02$ & 0.296 & & & \\
\hline \multicolumn{7}{|l|}{ Hypertension } \\
\hline No & Reference & & & & & \\
\hline Yes & 1.24 & $0.66-2.34$ & 0.503 & & & \\
\hline \multicolumn{7}{|l|}{$\mathrm{ACCl}$} \\
\hline$<5$ & Reference & & & & & \\
\hline$\geq 5$ & 1.75 & $0.96-3.19$ & 0.067 & & & \\
\hline
\end{tabular}

Table 2 (continued) 
Table 2 (continued)

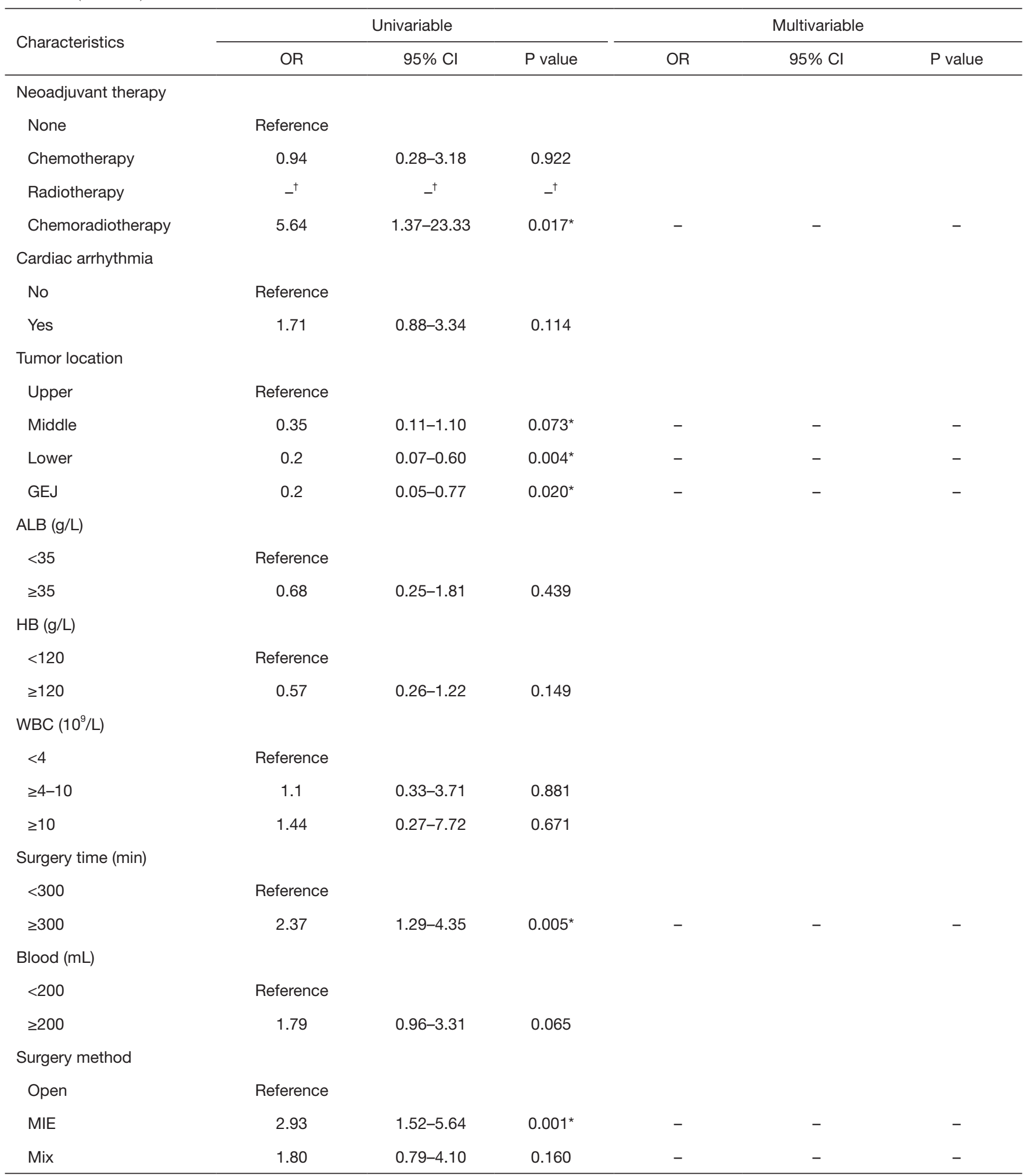

Table 2 (continued) 
Table 2 (continued)

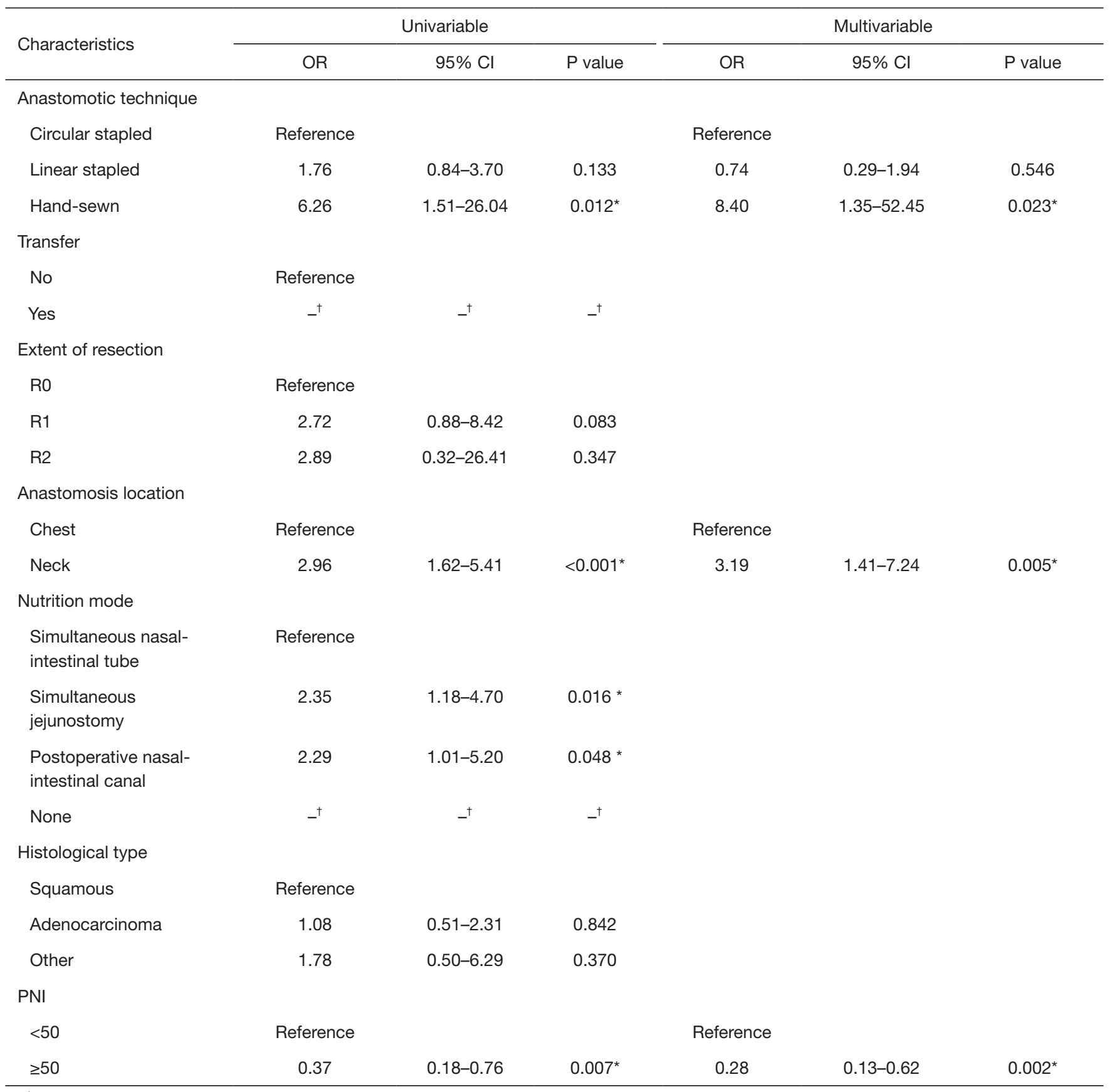

$-^{\dagger}$, The model failed because of the small sample size; *, P<0.05. SI, smoking index; BMI, body mass index; NRS, nutrition risk screening; ASA, American Society of Anesthesiologists; ACCI, age-adjusted Charlson comorbidity index; GEJ, gastroesophageal junction; ALB, albumin; Hb, hemoglobin; WBC, white blood cell; PNI, prognostic nutritional index. 


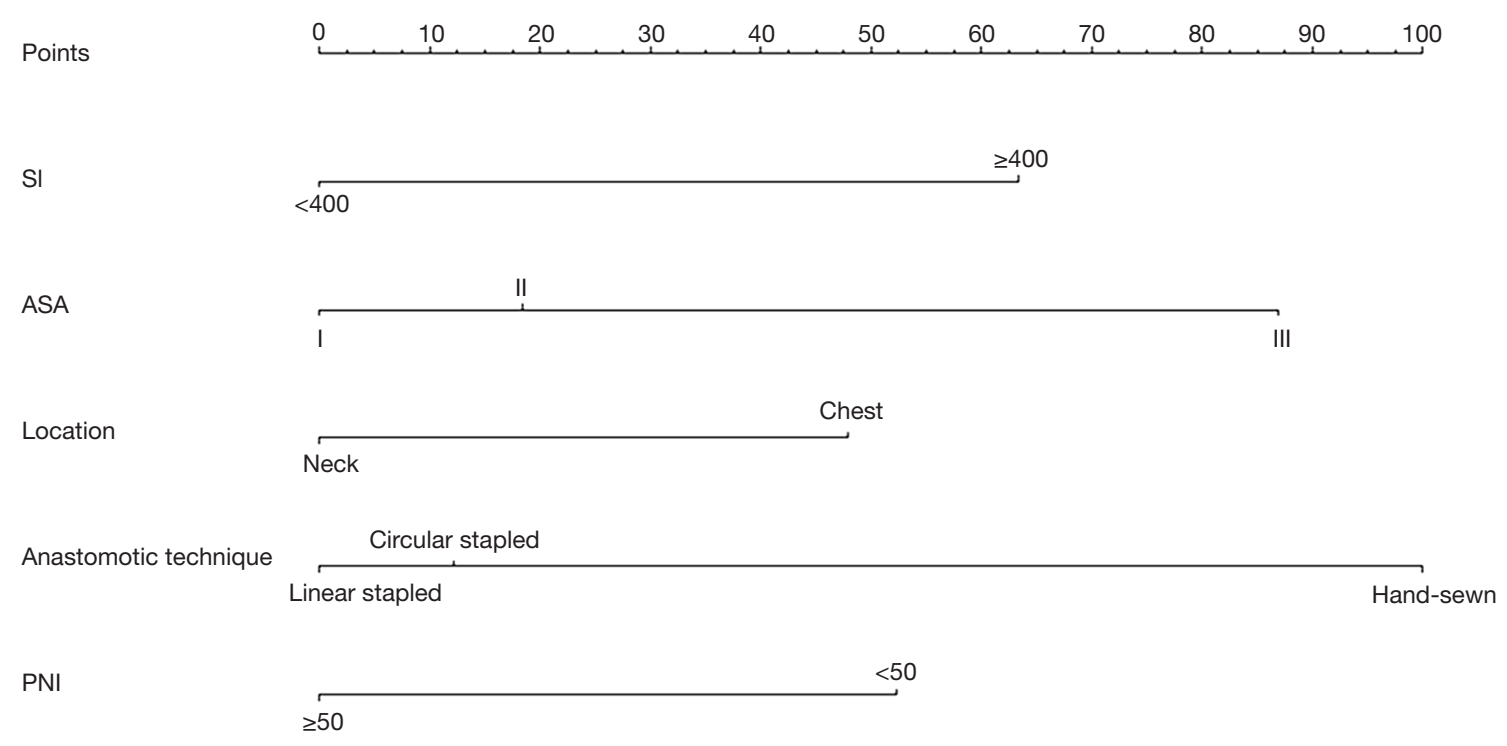

Total points

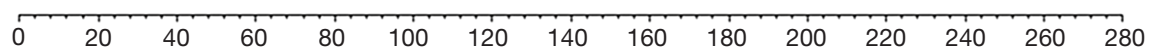

Risk of AL

$\begin{array}{lll}0.1+0.3 & 0.7\end{array}$

Figure 2 The nomogram to predict the risk of anastomotic leakage after esophagectomy. To use the nomogram, an individual patient's value is located on each variable axis, and a line is drawn upward to determine the number of points received for each variable value. The sum of these numbers is located on the Total Points axis, and a line is drawn downward to the risk axes to determine the likelihood of anastomotic leakage occurred after esophagectomy. SI, smoking index; PNI, prognostic nutritional index; ASA, American Society of Anesthesiologists; $\mathrm{AL}$, anastomotic leakage.

patients after esophagectomy, which assisted to develop the individualized therapeutic strategy.

Comparison of different studies has been long limited because of the lack of standardized definitions of post-esophagectomy complications. The definition of complications after esophagectomy proposed by ECCG has been increasingly used in clinical practice $(14,22)$, which was referenced in this study as well. As the benchmarking study of ECCG showed, the total incidence of AL is about $11.1 \%$, including $3.3 \%$ of Type I AL, $4.8 \%$ of Type II, and $3.0 \%$ of Type III (14). Consistently, the ratio of Type II $\mathrm{AL}$ cases remained the highest in our cohort. Therapeutic strategies of AL are determined by the size and statue of the fistula, but the treatment strategy is gradually changing from surgery to more conservative method (4). As a result, the number of Type III AL cases was relatively small. There were 4 patients receiving surgery because of early stage fistula or sepsis, and the remaining were cured after conservative treatment.
ASA score system is a simple, six-grade (I-VI) scale that rapidly classifies patients based on their overall health statue and comorbid conditions, which is used to predict the morbidity and mortality of surgical patients (23). ASA score has been confirmed as an independent risk factor of AL after colorectal cancer surgery (24). Whether ASA score is the risk factor of AL after esophagectomy is unclear. Consistent with our study, Gooszen et al. conducted a multivariate analysis on 654 cases of postoperative esophageal cancer patients, and they obtained the conclusion that ASA $>2$ is the independent risk factor of postoperative AL (20), but opposite results are also obtained in another study (22). Collectively, ASA score dose reflects the general physical condition of a patient to some extent. An inconsistent conclusion about ASA score may be attributed by the heterogeneity of data.

It has been widely verified that cervical anastomosis is more prone to develop AL than intrathoracic anastomosis. A meta-analysis involving data from 13 centers revealed 
Table 3 Point assignment and prognostic score

\begin{tabular}{lc}
\hline Variable & Prognostic score \\
\hline SI & 0 \\
$<400$ & 63.4 \\
$\geq 400$ & \\
ASA & 0 \\
I & 18.4 \\
II & 86.8 \\
III & \\
Location & 0 \\
Chest & 47.9 \\
Neck & \\
Anastomotic technique & \\
Linear stapled & 12.2 \\
Circular stapled & 100 \\
Hand-sewn & \\
PNI & \\
$\geq 50$ & \\
$<50$ & \\
\hline & \\
\hline
\end{tabular}

The scores corresponding to different levels of each predictor, which is convenient for calculating the total score of each patient. SI, smoking index; ASA, American Society of Anesthesiologists; PNI, prognostic nutritional index. For scoreprojected estimation, please see Figure 2.

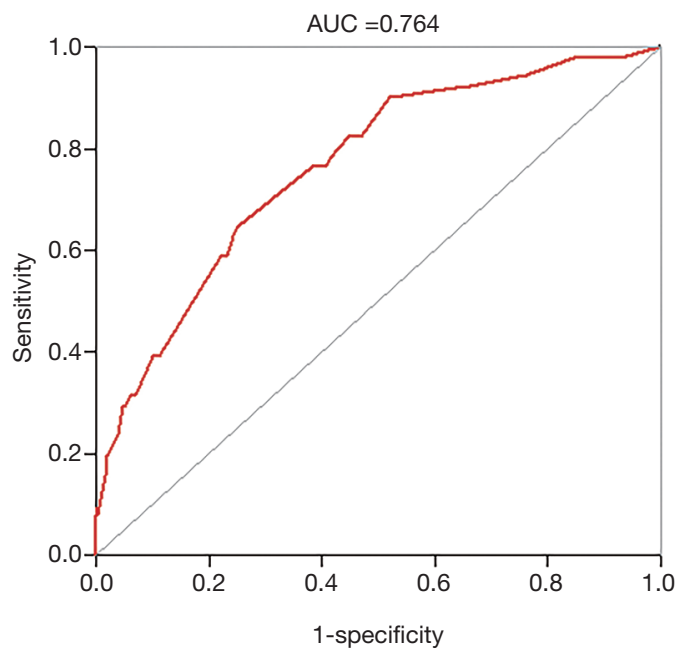

Figure 3 The ROC curve was established. The AUC was used to evaluate the discrimination of the model. ROC, receiver operating characteristic; AUC, area under the curve.

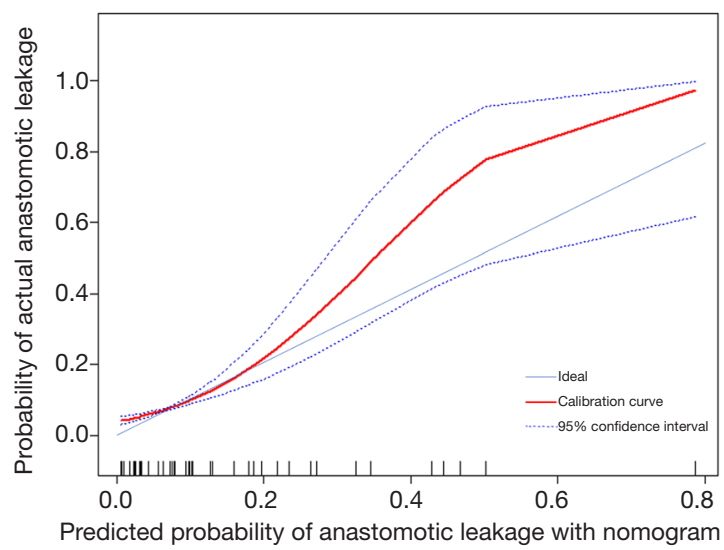

Figure 4 Calibration curve of nomograph. The red line represents the actual incidence of anastomotic leakage at different predictive probabilities. The blue solid line represents the situation where the predicted probability is exactly the same as the actual probability. In fact, it describes the fitting between the predicted probability and the actual probability, which is the calibration of the model.

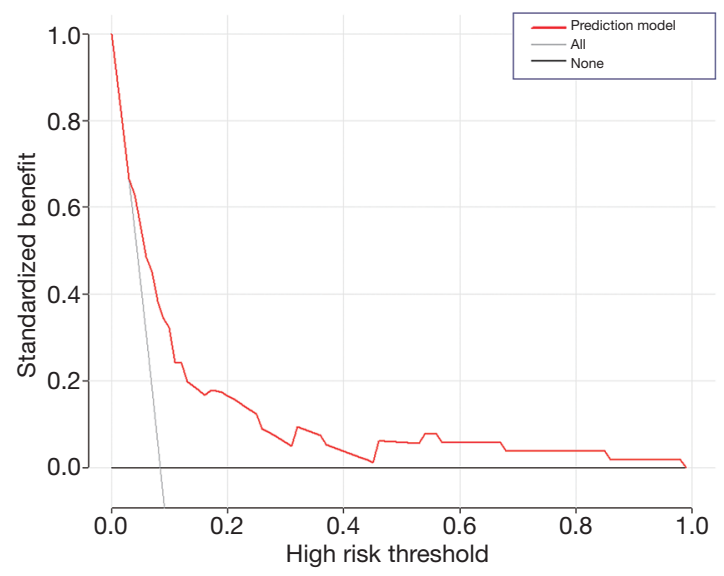

Figure 5 Decision curve for nomogram model to predict the risk of postoperative anastomotic leakage in patients with esophageal cancer. The graph shows the clinical net benefit of patients with anastomotic leakage defined by different threshold probabilities. Dotted line: assume all patients have anastomotic leakage. Grey thin line: assume no patients have anastomotic leakage. 
a higher incidence of AL in cervical anastomosis cases than others (pooled odds ratio $=4.73,95 \%$ CI, 1.61-13.9, $\mathrm{P}=0.005$ ) (25). Similar results are obtained in another metaanalysis involving 4 studies (26). It is generally considered that long stomach tubes required for the construction of cervical anastomosis, inadequate blood perfusion around the anastomotic site, large tension at cervical anastomosis, and large compression on cervical anastomosis because of superficial anatomic location of the neck all contribute to the enhanced incidence of AL in cervical anastomosis cases. Fortunately, these cases are easily to be detected in the early stage and managed owing to the superficial anatomic location of the neck. Overall, ICU stay, hospital stay and in-hospital mortality are not affected by anastomosis location (27). Compared with cervical AL, intrathoracic $\mathrm{AL}$ results in prolonged length of stay in ICU/hospital and delayed oral intake time. More seriously, over $50 \%$ of patients with chest $\mathrm{AL}$ require transthoracic re-intervention (open surgery or thoracoscopy), indicating that chest anastomosis causes a more severe clinical course (28).

So far, which anastomosis techniques can minimize the incidence of AL is controversial. Some evidences supported that hand-sewn, compared with mechanical anastomosis, would not increase the incidence of $\mathrm{AL}(4,29)$. On the contrary, multiple studies demonstrated that hand-sewn dose result in a higher incidence of AL than that of using linear or circular stapler $(30,31)$, because mechanical anastomosis is simply operated and causes a small tension and damage to tissues. Besides, manual anastomosis is largely affected by medical experiences of the operator.

In univariate analysis, AL was observed in 25 of the 182 MIE patients, and the statistical difference is significant. However, further multivariate analysis showed that MIE is not a risk factor for AL. Moreover, most studies have shown that there is no statistical difference between surgical methods and anastomotic leakage (4). In some studies, MIE surgery may cause more anastomotic leakage, which may require a certain learning curve due to the complexity of the operation (22). Smoking is a well-recognized risk factor that influences surgical healing, and its relationship with anastomotic leakage has been reported in esophageal surgery and other gastrointestinal surgery $(11,32)$. However, the mechanism of the effect of smoking on anastomotic leakage remains unclear. Previous evidences have shown that smoking decreases tissue oxygenation, blood perfusion, and the inflammatory healing reaction can be weakened by reducing the chemotaxis, migration function and oxidative sterilization mechanism of inflammatory cells (33). In addition, smoking also attenuates the synthesis of collagens, and proliferation and migration of fibroblasts, thus impairing the proliferative response. Quitting smoking for 4 weeks can partially alleviate the inflammatory cell response, but its effect on improving the proliferative response is limited (33). Considering adverse effects of smoking in the perioperative period, quitting smoking is encouraged at any moment.

The nutritional status of the patient is correlated to surgical risk, which significantly influences the healing process after surgery. In 1980, Buzby et al. designed PNI to assess potential risk of gastrointestinal surgery (34), which has been modified by Japanese scholars to use in assessing the nutritional status of other operated cancer patients gradually. Studies have confirmed that PNI is an independent risk factor of $\mathrm{AL}$ after gastric cancer surgery (35), and associated with clinical outcomes in esophageal cancer (36). Our findings have further proved that PNI is the independent risk factor of AL after esophagectomy.

In addition, no other risk factors associated with anastomotic leakage were found in our study. In fact, most of risk factors of AL after esophagectomy are still uncertain (4), although many of them seem to be related to AL. The nomogram established based on preoperative and perioperative data of patients could help physicians make better clinical decisions, identify and treat patients with anastomotic leakage earlier, and take preventive measures before clinical symptoms. For patients whose nomograms predict high AL risk, we can appropriately extend the start of oral feeding time (37), gastrointestinal decompression time, and strengthen postoperative nutritional support. After surgery, these patients will be tested more closely, including basic vital signs, whether they occur atrial fibrillation (38), daily assessment of the patient's inflammatory indicators and the amylase content in the closed thoracic drainage tube, etc. For patients with high suspicion of anastomotic leakage, barium swallow testing and endoscopy as soon as possible are helpful for early diagnosis and targeted treatment.

There were several limitations in this study. First of all, it was a single-center retrospective study that may cause potential biases. Secondly, because external validation cannot be implemented, we only performed an internal validation 1,000 bootstrap resample on the established nomogram. But our center, as a large regional diagnosis and treatment center, radiates tens of millions of people in a province, so we can infer that our results may have a certain degree of universality. In the future, we will start this work 
to further externally verify the model. Thirdly, because the purpose of this study was to predict postoperative anastomotic leakage, postoperative parameters such as pathological stage and pneumonia were not included. And some potential factors related to $\mathrm{AL}$, such as drug history, were also not included. Our findings require to be further validated in the future.

\section{Conclusions}

The study demonstrated that smoking index, anastomotic location, anastomotic technique, PNI and ASA score are independent risk factors for AL. In addition, this nomogram provided the individual prediction of anastomotic leakage for esophageal cancer patients after surgery, which might benefit treatment results for patients and clinicians, as well as pre- and postoperative intervention strategy-making.

\section{Acknowledgments}

Funding: None.

\section{Footnote}

Reporting Checklist: The authors have completed the TRIPOD reporting checklist. Available at http://dx.doi. org/10.21037/jtd-21-209

Data Sharing Statement: Available at http://dx.doi. org/10.21037/jtd-21-209

Conflicts of Interest: All authors have completed the ICMJE uniform disclosure form (available at http://dx.doi. org/10.21037/jtd-21-209). The authors have no conflicts of interest to declare.

Ethical Statement: The authors are accountable for all aspects of the work in ensuring that questions related to the accuracy or integrity of any part of the work are appropriately investigated and resolved. The study was conducted in accordance with the Declaration of Helsinki (as revised in 2013). The study was approved by the ethics committee of Affiliated Hospital of Qingdao University (NO.: QYFYWZLL26314). This study was a retrospective study. All patients signed the informed consent for operation before the operation, and no need for informed consent again.
Open Access Statement: This is an Open Access article distributed in accordance with the Creative Commons Attribution-NonCommercial-NoDerivs 4.0 International License (CC BY-NC-ND 4.0), which permits the noncommercial replication and distribution of the article with the strict proviso that no changes or edits are made and the original work is properly cited (including links to both the formal publication through the relevant DOI and the license). See: https://creativecommons.org/licenses/by-nc-nd/4.0/.

\section{References}

1. Ajani JA, D'Amico TA, Bentrem DJ, et al. Esophageal and Esophagogastric Junction Cancers, Version 2.2019, NCCN Clinical Practice Guidelines in Oncology. J Natl Compr Canc Netw 2019;17:855-83.

2. Seesing MFJ, Gisbertz SS, Goense L, et al. A Propensity Score Matched Analysis of Open Versus Minimally Invasive Transthoracic Esophagectomy in the Netherlands. Ann Surg 2017;266:839-46.

3. Chen C, Yu Z, Jin Q, et al. Clinical features and risk factors of anastomotic leakage after radical esophagectomy. Zhonghua Wai Ke Za Zhi 2015;53:518-21.

4. Fabbi M, Hagens ERC, van Berge Henegouwen MI, et al. Anastomotic leakage after esophagectomy for esophageal cancer: definitions, diagnostics, and treatment. Dis Esophagus 2021;34:doaa039.

5. Fransen LFC, Berkelmans GHK, Asti E, et al. The Effect of Postoperative Complications After Minimally Invasive Esophagectomy on Long-term Survival: An International Multicenter Cohort Study. Ann Surg 2020. [Epub ahead of print]. doi: 10.1097/SLA.0000000000003772.

6. Rutegård M, Lagergren P, Rouvelas I, et al. Intrathoracic anastomotic leakage and mortality after esophageal cancer resection: a population-based study. Ann Surg Oncol 2012;19:99-103.

7. Markar S, Gronnier C, Duhamel A, et al. The Impact of Severe Anastomotic Leak on Long-term Survival and Cancer Recurrence After Surgical Resection for Esophageal Malignancy. Ann Surg 2015;262:972-80.

8. Kassis ES, Kosinski AS, Ross P, Jr., et al. Predictors of anastomotic leak after esophagectomy: an analysis of the society of thoracic surgeons general thoracic database. Ann Thorac Surg 2013;96:1919-26.

9. Price TN, Nichols FC, Harmsen WS, et al. A comprehensive review of anastomotic technique in 432 
esophagectomies. Ann Thorac Surg 2013;95:1154-60; discussion 1160-1.

10. Goense L, van Rossum PSN, Ruurda JP, et al. Radiation to the Gastric Fundus Increases the Risk of Anastomotic Leakage After Esophagectomy. Ann Thorac Surg 2016;102:1798-804.

11. Cooke DT, Lin GC, Lau CL, et al. Analysis of cervical esophagogastric anastomotic leaks after transhiatal esophagectomy: risk factors, presentation, and detection. Ann Thorac Surg 2009;88:177-84; discussion 184-5.

12. Zafirellis KD, Fountoulakis A, Dolan K, et al. Evaluation of POSSUM in patients with oesophageal cancer undergoing resection. Br J Surg 2002;89:1150-5.

13. Haga Y, Wada Y, Takeuchi H, et al. Prediction of anastomotic leak and its prognosis in digestive surgery. World J Surg 2011;35:716-22.

14. Low DE, Kuppusamy MK, Alderson D, et al. Benchmarking Complications Associated with Esophagectomy. Ann Surg 2019;269:291-8.

15. Pennathur A, Gibson MK, Jobe BA, et al. Oesophageal carcinoma. Lancet 2013;381:400-12.

16. Ueda Y, Shiraishi N, Toujigamori M, Shiroshita H, Etoh T, Inomata M. Laparoscopic Proximal Gastrectomy With Gastric Tube Reconstruction. JSLS 2016;20:e2016.00046.

17. Schisterman EF, Perkins NJ, Liu A, et al. Optimal cutpoint and its corresponding Youden Index to discriminate individuals using pooled blood samples. Epidemiology 2005;16:73-81.

18. Alba AC, Agoritsas T, Walsh M, et al. Discrimination and Calibration of Clinical Prediction Models: Users' Guides to the Medical Literature. Jama 2017;318:1377-84.

19. Vickers AJ, Elkin EB. Decision curve analysis: a novel method for evaluating prediction models. Med Decis Making 2006;26:565-74.

20. Gooszen JAH, Goense L, Gisbertz SS, et al. Intrathoracic versus cervical anastomosis and predictors of anastomotic leakage after oesophagectomy for cancer. Br J Surg 2018;105:552-60.

21. Copeland GP, Jones D, Walters M. POSSUM: a scoring system for surgical audit. Br J Surg 1991;78:355-60.

22. Fumagalli U, Baiocchi GL, Celotti A, et al. Incidence and treatment of mediastinal leakage after esophagectomy: Insights from the multicenter study on mediastinal leaks. World J Gastroenterol 2019;25:356-66.

23. Kuza CM, Matsushima K, Mack WJ, et al. The role of the American Society of anesthesiologists physical status classification in predicting trauma mortality and outcomes. Am J Surg 2019;218:1143-51.
24. McDermott FD, Heeney A, Kelly ME, et al. Systematic review of preoperative, intraoperative and postoperative risk factors for colorectal anastomotic leaks. Br J Surg 2015;102:462-79.

25. Markar SR, Arya S, Karthikesalingam A, et al. Technical factors that affect anastomotic integrity following esophagectomy: systematic review and meta-analysis. Ann Surg Oncol 2013;20:4274-81.

26. Biere SS, Maas KW, Cuesta MA, et al. Cervical or thoracic anastomosis after esophagectomy for cancer: a systematic review and meta-analysis. Dig Surg 2011;28:29-35.

27. van Workum F, van der Maas J, van den Wildenberg FJ, et al. Improved Functional Results After Minimally Invasive Esophagectomy: Intrathoracic Versus Cervical Anastomosis. Ann Thorac Surg 2017;103:267-73.

28. van Rossum PSN, Haverkamp L, Carvello M, et al. Management and outcome of cervical versus intrathoracic manifestation of cervical anastomotic leakage after transthoracic esophagectomy for cancer. Dis Esophagus 2017;30:1-8.

29. Honda M, Kuriyama A, Noma H, et al. Hand-sewn versus mechanical esophagogastric anastomosis after esophagectomy: a systematic review and meta-analysis. Ann Surg 2013;257:238-48.

30. Deng XF, Liu QX, Zhou D, et al. Hand-sewn vs linearly stapled esophagogastric anastomosis for esophageal cancer: a meta-analysis. World J Gastroenterol 2015;21:4757-64.

31. Liu QX, Qiu Y, Deng XF, et al. Comparison of outcomes following end-to-end hand-sewn and mechanical oesophagogastric anastomosis after oesophagectomy for carcinoma: a prospective randomized controlled trial. Eur J Cardiothorac Surg 2015;47:e118-23.

32. Kim MJ, Shin R, Oh HK, et al. The impact of heavy smoking on anastomotic leakage and stricture after low anterior resection in rectal cancer patients. World J Surg 2011;35:2806-10.

33. Sørensen LT. Wound healing and infection in surgery: the pathophysiological impact of smoking, smoking cessation, and nicotine replacement therapy: a systematic review. Ann Surg 2012;255:1069-79.

34. Buzby GP, Mullen JL, Matthews DC, et al. Prognostic nutritional index in gastrointestinal surgery. Am J Surg 1980;139:160-7.

35. Oshi M, Kunisaki C, Miyamoto H, et al. Risk Factors for Anastomotic Leakage of Esophagojejunostomy after Laparoscopy-Assisted Total Gastrectomy for Gastric Cancer. Dig Surg 2018;35:28-34.

36. Okadome K, Baba Y, Yagi T, et al. Prognostic Nutritional 
Index, Tumor-infiltrating Lymphocytes, and Prognosis in Patients with Esophageal Cancer. Ann Surg 2020;271:693-700.

37. Speicher JE, Gunn TM, Rossi NP, et al. Delay in Oral Feeding is Associated With a Decrease in Anastomotic

Cite this article as: $\mathrm{Yu}$ WQ, Gao HJ, Shi GD, Tang JY, Wang HF, Hu SY, Wei YC. Development and validation of a nomogram to predict anastomotic leakage after esophagectomy for esophageal carcinoma. J Thorac Dis 2021;13(6):3549-3565. doi: 10.21037/jtd-21-209
Leak Following Transhiatal Esophagectomy. Semin Thorac Cardiovasc Surg 2018;30:476-84.

38. Seesing MFJ, Borggreve AS, Ruurda JP, et al. New-onset atrial fibrillation after esophagectomy for cancer. J Thorac Dis 2019;11:S831-4. 